\title{
Characteristics and outcomes of glomerulonephritis with membranoproliferative pattern in children
}

\author{
Linan $\mathrm{Xu}^{1,2,3 \#}$, Fengfang Wei" ${ }^{4 \#}$, Jiayan Feng ${ }^{5}$, Jiaojiao Liu ${ }^{1,2,3}$, Jialu Liu ${ }^{1,2,3}$, Xiaoshan Tang ${ }^{1,2,3}$, \\ Xiaoyan Fang ${ }^{1,2,3}$, Jing Chen ${ }^{1,2,3}$, Yihui Zhai ${ }^{1,2,3}$, Haimei Liu ${ }^{6}$, Li Sun $^{6}$, Yanyan Qian ${ }^{7}$, Bingbing Wu \\ Huijun Wang ${ }^{7}$, Qian Shen ${ }^{1,2,3}$, Jia Rao ${ }^{1,2,3} \wedge$, Hong $\mathrm{Xu}^{1,2,3}$
}

${ }^{1}$ Department of Nephrology, Children's Hospital of Fudan University, National Children's Medical Center, Shanghai, China; ${ }^{2}$ Shanghai Kidney Development and Pediatric Kidney Disease Research Center, Shanghai, China; ${ }^{3}$ Shanghai Key Lab of Birth Defect, Children's Hospital of Fudan University, Shanghai, China; ${ }^{4}$ Affiliated Hospital of Putian University, Fujian, China; ${ }^{5}$ Department of Pathology, Children's Hospital of Fudan University, Shanghai, China; ${ }^{6}$ Department of Rheumatology, Children's Hospital of Fudan University, Shanghai, China; ${ }^{7}$ Clinical Genetic Center, Children's Hospital of Fudan University, Shanghai, China

Contributions: (I) Conception and design: J Rao, L Xu; (II) Administrative support: H Xu, Q Shen, H Liu, L Sun; (III) Provision of study materials or patients: Y Qian, B Wu, H Wang, J Liu, X Tang, X Fang, J Chen, Y Zhai, J Liu; (IV) Collection and assembly of data: L Xu, F Wei, J Feng, J Liu; (V) Data analysis and interpretation: L Xu; (VI) Manuscript writing: All authors; (VII) Final approval of manuscript: All authors.

\#These authors contributed equally to this work and are the co-first authors.

Correspondence to: Hong Xu, MD, PhD. Department of Nephrology, Children's Hospital of Fudan University, National Pediatric Medical Center of China, 399 Wanyuan Road, Shanghai, China. Email: hxu@shmu.edu.cn; Jia Rao, MD, PhD. Department of Nephrology, Children’s Hospital of Fudan University, National Pediatric Medical Center of China, 399 Wanyuan Road, Shanghai, China. Email: jiarao@fudan.edu.cn.

Background: Membranoproliferative glomerulonephritis (MPGN) is a rare histopathologic pattern of glomerular injury with limited studies in pediatric patients. Characteristics and outcomes of children with MPGN have also remained to be further explored.

Methods: We retrospectively reviewed the clinicopathological features, genetic findings, treatments and outcomes in 17 pediatric patients pathologically diagnosed with MPGN from 2007 to 2020 in the Children's National Medical Center in China.

Results: Median age at disease onset was 9.9 years (IQR, 5.6-11.9 years). Most of the patients (12/17) had nephrotic range of proteinuria, and nephritic-nephrotic syndrome was the most common clinical presentation (35.2\%). Secondary causes were identified in eight patients including hepatitis B virus (HBV) infection ( $n=4)$, methylmalonic acidemia (MMA, n=2), rheumatoid arthritis (RA, n=1) and Aymé-Gripp Syndrome $(n=1)$. The nine patients with primary MPGN were further identified as immune-complex mediated MPGN ( $\mathrm{n}=8$ ), and unclassifiable MPGN (U-MGPN, n=1). Genetic analyses identified pathogenic variants of $M M A C H C$ gene in two cases of MMA and established the diagnosis for Aymé-Gripp syndrome in one case with a de novo variant of $M A F$ gene. Comparing study between the complete or partial remission group $(n=8)$ and non-response group $(n=9)$ showed a significant difference in the timing of renal biopsy $(\mathrm{P}<0.05)$. Normal renal function was preserved in ten patients at the last follow-up. Two patients developed into end-stage renal disease (ESRD).

Conclusions: Children with MPGN pattern present heterogenous clinical features. Genetic detection helps to explore underlying causes of MPGN. Early identification of the primary or secondary causes of MPGN in children is vital.

Keywords: Membranoproliferative glomerulonephritis; C3 glomerulopathy; children

Submitted Jun 20, 2021; Accepted for publication Aug 16, 2021.

doi: $10.21037 / \mathrm{tp}-21-286$

View this article at: https://dx.doi.org/10.21037/tp-21-286

$\wedge$ ORCID: 0000-0003-2839-3419. 


\section{Introduction}

Membranoproliferative glomerulonephritis (MPGN) has been recognized as a rare pathological pattern of glomerulopathy clinically characterized by proteinuria, hematuria, hypertension and often impaired renal function at disease onset. It accounts for approximately $1-7 \%$ of all cases of biopsy-confirmed glomerulonephritis among all ages (1-4). Recent studies had revealed the incidence of this pattern was significantly declined in the 21st century, however, rates of progression to ESRD and death remained unimproved $(4,5)$. In up to $50 \%$ of the affected children, MPGN leads to endstage renal disease (ESRD) within ten years (6).

The typical features of MPGN on light microscopy (LM) include mesangial cellularity, endocapillary proliferation, and capillary-wall remodeling (with the formation of double contours), and lobular accentuation of glomerular tufts. Based on the electron-microscopical findings, MPGN is traditionally classified as primary (idiopathic) MPGN type I (MPGN I, with subendothelial deposits), type II (MPGN II, with dense deposits in the glomerular basement membrane), or type III (MPGN III both subepithelial and subendothelial deposits) or secondary MPGN (7). However, this kind of assortment neither indicates the etiology of MPGN, nor provides competent evidence for subsequent treatments. Lately, abnormal activation of complement via alternative pathway was found to mediate the formation of MPGN pattern (8). In 2013, an expert consensus was established and proposed a practical approach to view MPGN as immune-complex-mediated MPGN (IC-MPGN) and C3 glomerulopathy (C3G) based on immunofluorescence (IF) of renal biopsies (9). C3G was recognized by the new classification as a distinct type from MPGN pattern and further subdivided into dense deposit disease (DDD) and C3 glomerulonephritis (C3GN), depending on the position of electron-microscopical deposits. Although IC-MPGN and C3G were distinguished in histopathology, the essential borderline and interrelation between these two entities are still ambiguous.

Several studies were done to evaluate the causes, clinical presentations, effects of various treatments and prognosis of adult MPGN (10-14), yet studies of children MPGN remain to be small-scale with narrow cases $(15-17)$. The rareness of the disease as well the terminology shift with the revolution of diagnostic classification conceal the authentic characteristics and outcomes from being concluded. Consequently, effective therapies of MPGN pattern and ameliorated prognosis have not been made further.
The current therapies for MPGN including steroids and immunosuppressants suggested by KDIGO guidelines (18) have not shown consistent benefits and the evidence for therapeutic efficacy in children was extremely limited. Moreover, the latest classification of IC-MPGN and C3G remained to be evaluated on its utility in children with MPGN. Therefore, we aim to retrospectively analyze the clinical, pathological and pathogenic diagnosis of MPGN in children to provide an optimized strategy for early diagnosis of MPGN. We present the following article in accordance with the STROBE reporting checklist (available at https:// dx.doi.org/10.21037/tp-21-286).

\section{Methods}

\section{Study design and participants}

The children with pathologic diagnosis as MPGN, aged from birth to 18 years old were enrolled at Children's Hospital of Fudan University between January 1, 2007 and May 31, 2020. Following informed consent, we collected clinical records among the individuals with MPGN. A retrospective analysis of clinical features, pathological findings, genetic detection and renal outcome was performed. The study was conducted in accordance with the Declaration of Helsinki (as revised in 2013). The study was approved by Institutional Review Board (IRB) of Children's Hospital of Fudan University (No. 2018_286) and informed consent was taken from all the patients' parents.

\section{Measurements and variable definitions}

The definition of clinical phenotype and remission was shown in Table 1. Clinical data included gender, age of onset, initial presentation, treatment and outcomes were summarized in Table 2. All biopsies were prepared by standard techniques for LM, IF, and electron microscopy (EM), where available. Hematoxylin and eosin (HE) stain and periodic acid-silver methenamine (PASM) stain were applied for LM. The intensity of immunofluorescent staining for IgG, IgA, IgM, C3, C4, C1q, C3d, C9 and $\mathrm{Fb}$ was graded from negative to $3+$ on IF. Distribution, type and extent of deposits were recorded on EM. All renal diseases were diagnosed based on the KDIGO guideline $(18,19)$. Clinicians were asked to review the medical records and kidney biopsy findings of patients diagnosed with MPGN followed up for at least 3 months. 
Table 1 Definition of clinical remission, manifestations and diagnosis groups

\begin{tabular}{|c|c|}
\hline Term & Interpretation \\
\hline Complete remission & 24-hour urinary protein $<0.2 \mathrm{~g}$ or negative/trace in dipstick \\
\hline Partial remission & $\begin{array}{l}\text { Reduction of proteinuria of more than } 50 \% \text { compared to highest value (reduction more than } \\
50 \% \text { of } 24 \text {-hour urinary protein, or decrease at least } 2 \text { order in dipstick) With or without } \\
\text { hematuria }\end{array}$ \\
\hline Non-remission & Lack of complete or partial response \\
\hline Nephrotic syndrome & Edema, proteinuria (24-hour urinary protein $>50$ mg/kg), hypoalbuminemia (<30 g/L) \\
\hline Nephritic syndrome & $\begin{array}{l}\text { Macroscopic hematuria, edema, hypertension, abnormal function } \\
\left(\text { eGFR }<90 \mathrm{~mL} / \mathrm{min} \cdot 1.73 \mathrm{~m}^{2}\right) \text {, proteinuria (24-hour urinary protein }>50 \mathrm{mg} / \mathrm{kg} \text { ) }\end{array}$ \\
\hline Nephritic-nephrotic syndrome & Joint occurrence of nephrotic and nephritic syndrome \\
\hline
\end{tabular}

C3G, C3 glomerulopathy; eGFR was calculated by the Schwartz formula using a local k-factor of 49 in CKD 1-2, 36.5 in CKD 3-5.

Biopsy findings of patients diagnosed with MPGN or post infectious glomerulonephritis before the publication of the new $\mathrm{C} 3 \mathrm{G}$ classification criteria were re-evaluated and patients meeting the new $\mathrm{C} 3 \mathrm{G}$ criteria were included in the study. All patients were subsequently classified as ICMPGN or C3G (including C3GN and DDD). The current criterion for $\mathrm{C} 3 \mathrm{G}$ is based on the IF criteria of predominant glomerular C3 intensity of $\geq 2$ levels of magnitude greater than any other immune reactant $(20,21)$. The definition of unclassifiable MPGN (U-MGPN) involves negative stating of complement or immunoglobin (partially due to poor quality staining) in renal species. Outcomes of patients were divided into complete remission group, partial remission group and non-response group (15). All patients were stratified by renal biopsy timing. The early diagnosis group was defined as biopsy performed within six months of the disease onset. Delayed diagnosis group was defined as biopsy performed over six months after the disease onset (Table 1).

\section{Statistical analysis}

Data were analyzed using Excel. Continuous variables were summarized with median, IQR and categorical data were summarized with proportions. Mann-Whitney $U$ test (for continuous variables that do not conform to the normal distribution and homoscedasticity) and the Fisher exact probability test (for categorical variables) were used to analyze the differences among participants with different clinical features stratified into early or delayed diagnosis groups. Spearman correlation analysis was performed to assess the correlation between serum $\mathrm{C} 3$ and proteinuria during the follow-up. Statistical analysis was performed with SPSS version 25.0 statistical package software (IBM Co., Armonk, NY, USA). Figures were performed using GraphPad Prism 7.0.

\section{Results}

A total of 17 children (ten boys and seven girls) with MPGN were recruited from the 1,901 (1.4\%) cases with complete clinical and pathological records of the children's hospital of Fudan University from 2007 to 2020. The median age at onset was 9.9 years (IQR, 5.6-11.9 years) with a median eGFR of $102.6 \mathrm{~mL} / \mathrm{min} \cdot 1.73 \mathrm{~m}^{2}$ (IQR, 47.0 $121.5 \mathrm{~mL} / \mathrm{min} \cdot 1.73 \mathrm{~m}^{2}$ ) at the time of admission. 


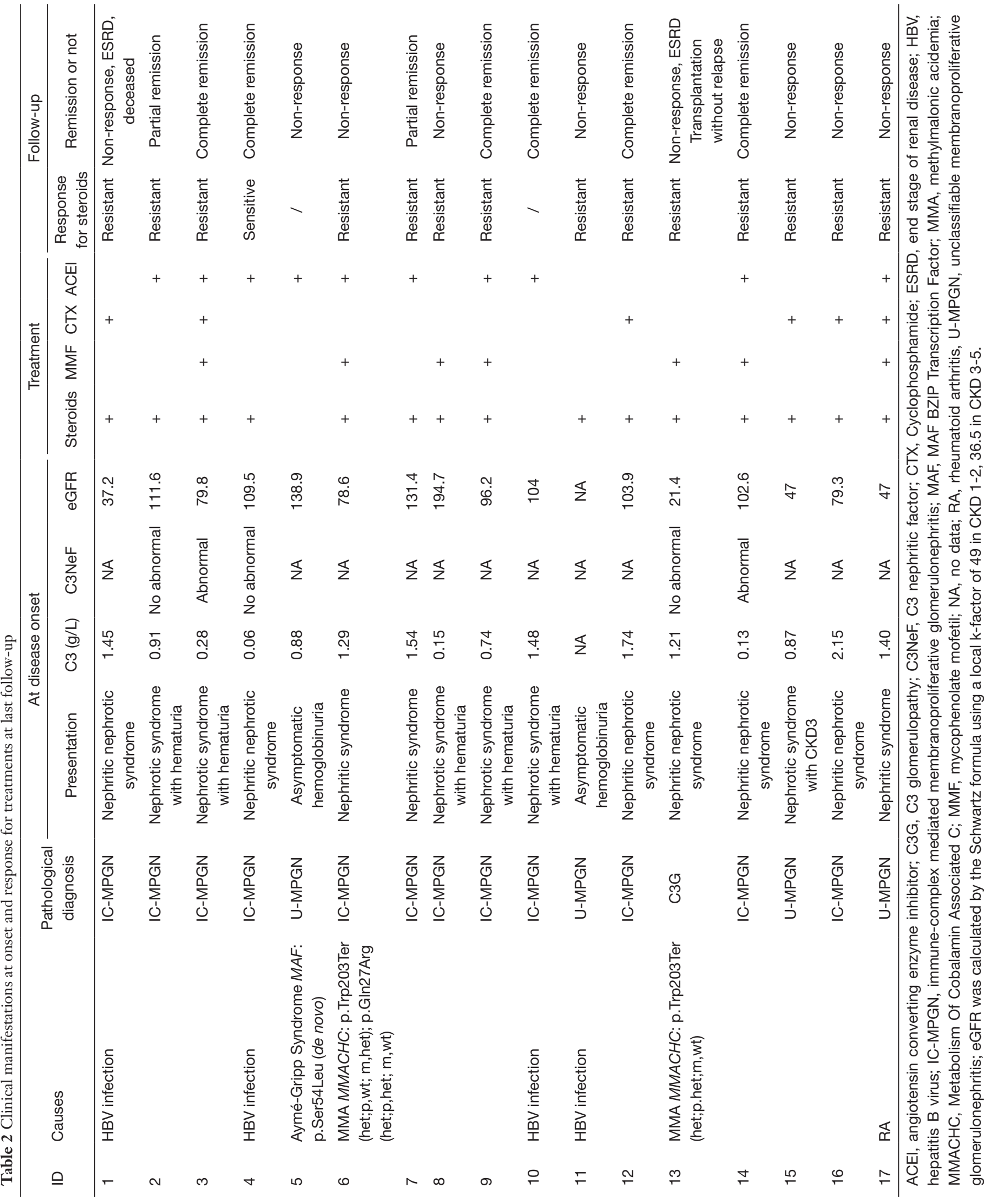




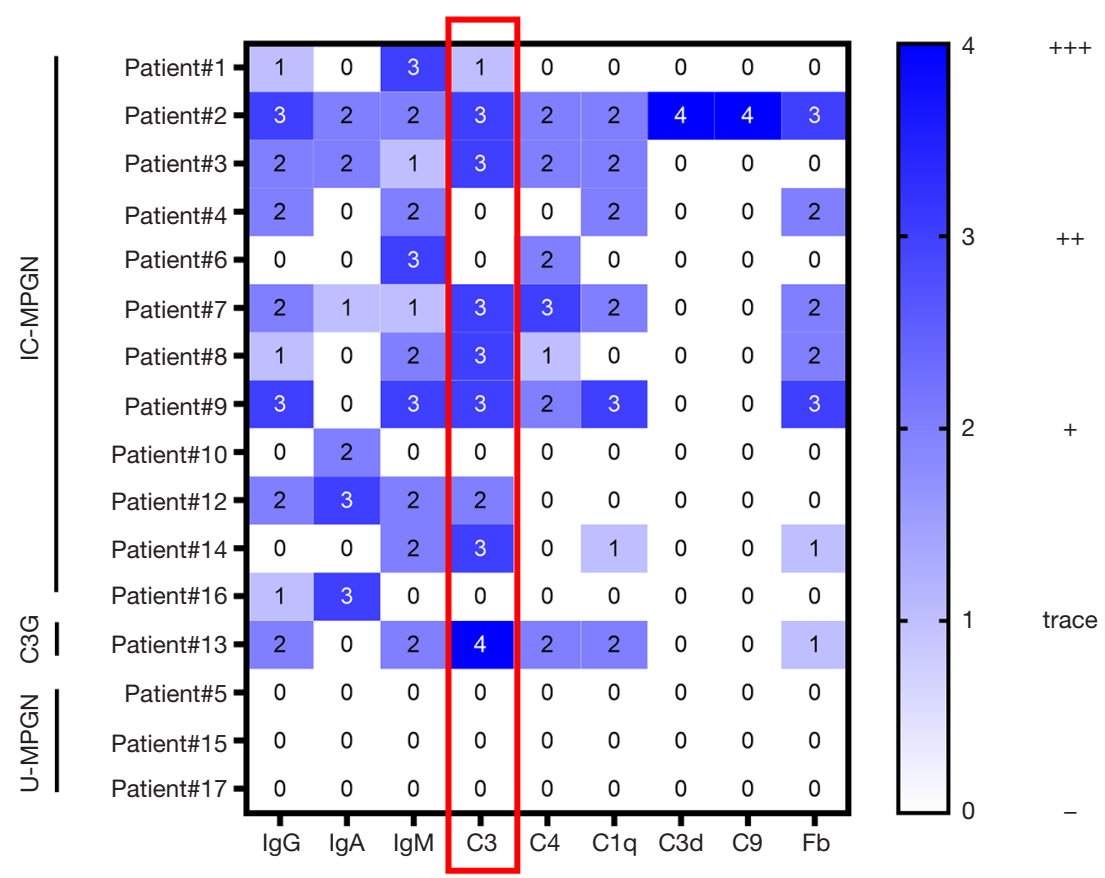

Figure 1 Deposits of immunoglobulin and complement in patients. Magnitude of intensity of immunofluorescent was suggested on a scale of 0 to 4 including negative, trace, $1+, 2+, 3+$. Immunofluorescence labeling of patient 11 failed because of inefficient biopsy, therefore, patient 11 was temporarily grouped into U-MPGN. C3G, C3 glomerulonephritis; IC-MPGN, immune-complex mediated membranoproliferative glomerulonephritis; U-MPGN, unclassifiable membranoproliferative glomerulonephritis.

\section{Clinical presentation at diagnosis and underlying causes}

Clinical and biological data for the patients at diagnosis are summarized in Table 2 and Table S1. Prior infections were reported in nearly half of the patients $(n=8)$, including upper respiratory tract infections $(n=4)$ and skin infections $(\mathrm{n}=4)$ (Table S1). In our study, most of the patients (12/17) had nephrotic range of proteinuria, and nephritic-nephrotic syndrome was the most common clinical presentation (Table 2). Fifteen $(88.2 \%, 15 / 17)$ patients had microscopic or macroscopic hematuria at onset. Hypocomplementemia was present in six $(35.3 \%$, 6/17) children including isolated low serum C3 values $(n=4)$, isolated low serum $C 4$ value $(n=1)$, and low serum $\mathrm{C} 3$ and $\mathrm{C} 4$ together $(\mathrm{n}=1)$. Hypertension and decline in kidney function were observed in four and seven patients at onset, respectively. Four patients had a history of familial glomerulonephritis (Table S2).

Underlying causes were found in a high proportion (8/17, 47.1\%) of our MPGN children (Table 2). Diagnosis of IC-MPGN makes up a larger proportion in our patients (12/17), and no DDD case was found in our patients, which is quite different from other studies mainly comprised by C3G (15), especially DDD (14). It may due to the high incidence of hepatitis $B$ virus infection in China. Four (23.5\%) patients were found to be secondary to hepatitis $\mathrm{B}$ virus infection and most of them presented with IC-MPGN. In addition to hepatitis B virus infection, MMA is another cause of MPGN. Two patients were diagnosed with MMA based on genetic analysis when developing into chronic kidney disease (CKD), and the prognosis was not as good as the other children. Standard therapy of vitamin B12 injections in responsive MMA patients should be started at an early stage. It suggests that more attention to paid to screening for MMA in childhood MPGN.

\section{Pathological findings and pathogenic diagnosis}

IF staining of renal biopsies was summarized in Figure 1. Three patients with HBV-associated MPGN showed ICMPGN, and one patient with $\mathrm{HBV}$-associated MPGN showed U-MPGN. One patient with MMA presented as IC-MPGN, and the other one with MMA showed C3G. 
One patient with rheumatoid arthritis (RA) was recorded as U-MPGN. Base on the current criterion, only one patient (patient \#13) secondary to MMA was diagnosed as $\mathrm{C} 3 \mathrm{G}$ with $\mathrm{C} 3$ dominance of two orders of magnitude stronger than any other immune reactant (20). However, we failed to establish the diagnosis of $\mathrm{C} 3 \mathrm{G}$ for the other four patients (patient \#3, $\# 7, \# 8$ and \#14) with C3 dominance of one order intensity greater than any other immune reactant by IF staining of renal biopsies. Three of the four patients had obvious activation of the alternative pathway with an abnormal level of $\mathrm{C} 3$ nephritic factor $(\mathrm{C} 3 \mathrm{NeF})$ or $\mathrm{C} 3$ (Table S2). None was diagnosed as DDD based on electron density deposition under the electron microscope. Therefore, the nine patients with primary MPGN were further identified as IC-MPGN ( $\mathrm{n}=8)$ and unclassifiable MPGN (U-MGPN, $\mathrm{n}=1$ ) (Table 2). Repeated renal biopsy was performed in patient \#3 because of relapse.

At initial presentation, low serum C3 values (normal $0.67-1.76 \mathrm{~g} / \mathrm{L})$ were presented in patients with $\mathrm{C} 3 \mathrm{G}(\mathrm{n}=1)$ and IC-MPGN (n=4) (Table 2). Screening for the presence of $\mathrm{C} 3 \mathrm{NeF}$ was performed in five patients. Abnormal level of $\mathrm{C} 3 \mathrm{NeF}$ were reported in two patients at onset (Table 2). Exome sequencing was performed in five patients considering the extrarenal phenotypes and $\mathrm{C} 3$ dominant deposit by immunofluorescence staining of renal biopsies with suspicion of congenital complement disorder. No pathogenic variations were identified in the complement regulatory genes. Compound heterogeneous variants of $M M A C H C$ were identified in one of the patients who were clinical suspicion of MMA with homocysteinemia.

One patient (patient \#5) was diagnosed with deafness, bilateral cataracts and intellectual disability within one year old who present non-nephrotic range proteinuria at age of eight years old. Serum creatinine was $26 \mu \mathrm{mol} / \mathrm{L}$, albumin $42.1 \mathrm{~g} / \mathrm{L}, \mathrm{C} 3$ was $0.88(0.67-1.76 \mathrm{~g} / \mathrm{L})$. Serologically he had negative ANA, pANCA/cANCA and dsDNA. Ultrasonography showed the normal size of kidneys with maintained cortical medullary differentiation. Kidney biopsy revealed MPGN without any deposits of immune reactant by immunofluorescence or electron microscope (Figure 2). In view of the multisystem phenotypes, we performed trio-exome sequencing identifying a de novo heterogeneous variant (p.Ser54Leu) of MAF gene which is the known molecular cause for Aymé-Gripp syndrome.

\section{Treatments and outcomes}

The median follow-up was 2.4 years (IQR, 1.0-4.5 years).
Nearly half $(47.3 \%, 8 / 17)$ of them were followed more than 3 years. We can see a high proportion of patients (14/15, $93.3 \%$ ) did not respond to the initial therapy of steroids. Therefore, additional immunosuppressive regimens were used in most of these patients to induce clinical remission (Table 2). The combination of steroids with MMF or CTX induced complete remission in four patients with poor response to initial steroids therapy. Complications of treatments were observed in eight patients during the follow-up, including hypertension $(\mathrm{n}=3)$, dental ulcer $(\mathrm{n}=2)$, cataract $(\mathrm{n}=1)$, blurred vision $(\mathrm{n}=1)$, high intraocular pressure $(n=1)$ and urinary tract infection $(n=3)$. Some patients had more than one complication. All the details could be found in Table S2.

At the last follow-up, nearly half of the patients responded well to the interventions and eight of them developed complete or partial remission (Figure 3). Although receiving aggressive therapies, two patients progressed to ESRD 10.5 months and 4.7 years after disease onset respectively, and one of them deceased within a year post ESRD. Since nearly half of our patients with MPGN do not respond well to routine treatments, it is important to explore the factors associated with renal prognosis. We analyzed clinical factors including the etiology, disease onset age, treatments, family history, the timing of pathological diagnosis and extrarenal manifestations (Table 3). There were more patients who achieve full or partial remission in the early diagnosis patient group compared with the patients from the delayed diagnosis patient group $(\mathrm{P}<0.05$, Table 3). The relationship between the timing of renal biopsy and outcomes were further marked in Figure 3. All patients received early diagnosis had achieved a complete or partial remission at the last follow-up, except for those patients with secondary MGPN caused by MMA and Aymé-Gripp Syndrome.

Although consistent reduced serum C3 value was considered as a sign for activation of the alternative complement pathway, the relationship between serum C3 level and proteinuria in MPGN patients was still undetermined. Therefore, we analyzed the correlation between serum C3 levels and 24-hour urinary protein during the follow-up of two patients (patient \#3 and \#14) with positive $\mathrm{C} 3 \mathrm{NeF}$ and detailed information during followup (Figure 4). Patient \#3 presented with nephrotic-range proteinuria, hematuria and abnormal kidney function at disease onset (Table S1). Complement tests showed a low level of serum C3 levels and elevated C3NeF. Steroids and RAAS blockers were started. A partial remission with decreasing proteinuria was showed, whereas the $\mathrm{C} 3$ level 

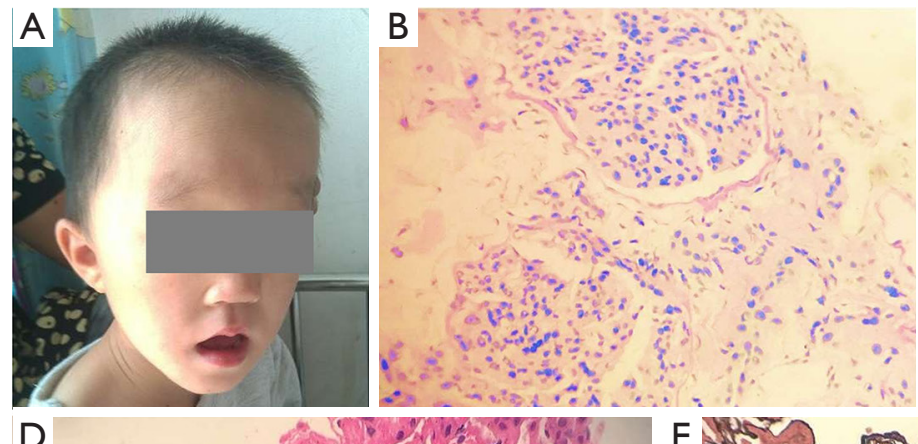

C
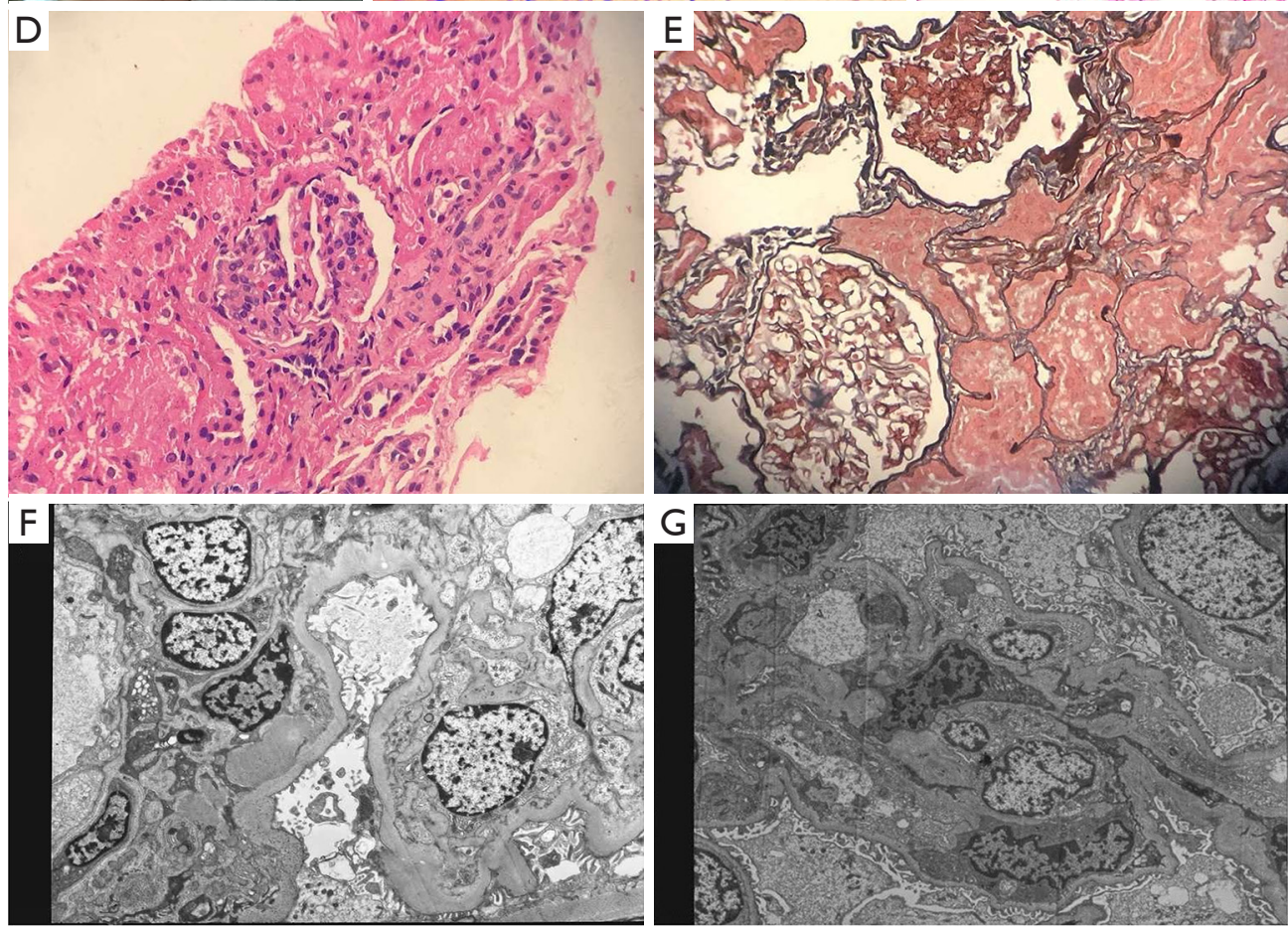

Figure 2 Kidney of patient \#5 with Aymé-Gripp Syndrome. (A) Patient \#5 manifests congenital cataracts, sensorineural hearing loss, intellectual disability and distinctive flat facial appearance. This image is published with the patient's consent. (B-D) Glomeruli with mesangial and endocapillary proliferation and interstitial fibrosis (hematoxylin and eosin stain, original magnification $\times 200$ ). (E) Moderate to severe mesangial proliferation (periodic acid-silver methenamine stain, original magnification $\times 200$ ). (F,G) Electron microscopy with renal afferent arteriolar dense deposit but no osmophilia electron-dense deposits in the lamina dense of the glomerular basement membrane and mycangium (original magnification $\times 1,000, \times 1,200$ ).

remained low after eight weeks of steroids therapy. Because of recurrent proteinuria when steroids tapering accompanied by a skin infection, mycophenolate mofetil (MMF) was started in combination with steroids. After a twenty-month remission, proteinuria subsequently relapsed again when reducing the dosage of MMF. Then, MMF was stopped and shifted to cyclophosphamide (CTX). Eight months after starting CTX, a significant decline of proteinuria and increased serum C3 were observed with stabilization of eGFR. Patient \#14 presented with nephritic-nephrotic syndrome and normal renal function at the onset. After a poor response to steroids initially, she started MMF. Complete remission of proteinuria and maintained normal renal function were achieved with successfully tapering steroids and MMF, whereas serum C3 remained persistently low value. We can see a negative relationship between serum $\mathrm{C} 3$ value and urine protein (Figure 4), whereas serum $\mathrm{C} 3$ value and $\mathrm{C} 3 \mathrm{NeF}$ level was not found correlatedly (Figure S1). 


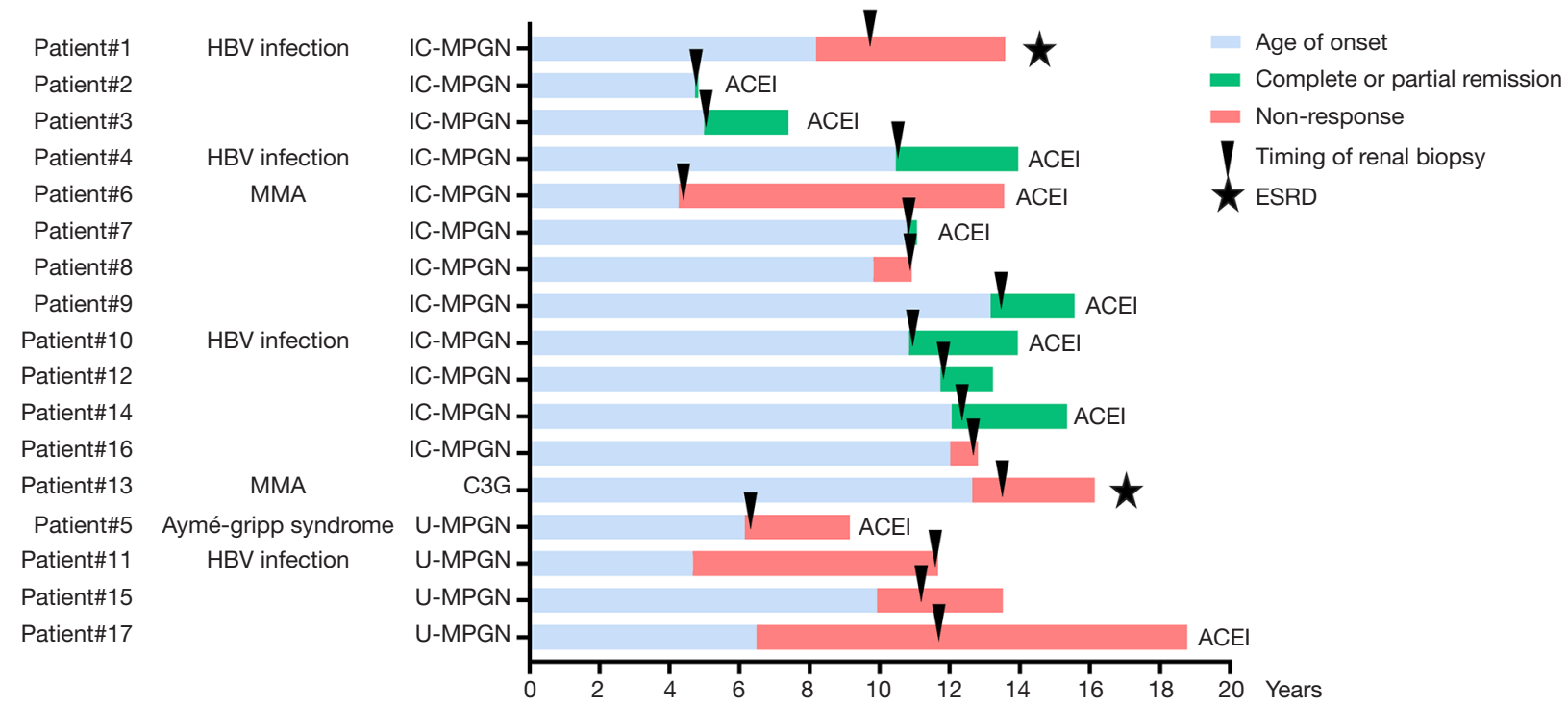

Figure 3 Timing of pathological diagnosis based on renal biopsy and the correspondent clinical remission of MPGN patients. ACEI, angiotensin converting enzyme inhibitor; C3G, C3 glomerulopathy; ESRD, end-stage of renal disease; HBV, hepatitis B virus; IC-MPGN, immune-complex mediated membranoproliferative glomerulonephritis; MMA, methylmalonic acidemia; RA, rheumatoid arthritis.

\section{Discussion}

This study presented the clinical features of 17 children with MPGN of kidney injury in a single center in China providing evidences for diagnosis and outcomes for childhood MPGN. It is essential to identify the diagnosis in MPGN concerning the pathological classification, secondary etiology and genetic causes. A previous study had reported lupus nephritis was the most common cause among adult patients with secondary MPGN (12), and our results presented $\mathrm{HBV}$ infection as the most common cause for secondary MPGN in pediatric patients. A heterogenous clinical presentation of our patients displayed as nephriticnephrotic syndrome, nephrotic syndrome with hematuria or abnormal renal function, nephritic syndrome and asymptomatic hematuria or proteinuria, in line with the previous reports $(22,23)$.

Genetic detection helps to explore the underlying causes of MPGN. The invariable presence of $\mathrm{C} 3$ in the glomerulus has implicated complement alternative pathway activation as a key causal mechanism and testing for complement gene mutations is currently recommended in C3G. It has been documented by a study of 146 European cases and 6,442 European controls that there is no association of rare complement gene variants with primary MPGN (24). Although we did not observe rare pathological variants in the candidate genes (encoding components of the complement alternative pathway), further analysis on common alleles of the other genes may show possible causal mechanisms for MPGN. Besides the genetic findings in $M M A C H C$ gene to support the diagnosis of MMA, we also identify the de novo variant of MAF gene (p.Ser54Leu) for Aymé-Gripp syndrome. Dominant pathogenic variants of MAF (MIM\# 601088) are associated with Aymé-Gripp syndrome, a condition presenting with mild to severe intellectual disability syndrome, autism spectrum disorder, cataracts, short stature, seizures, and skeletal involvement. To date, there were only two adult cases of Aymé-Gripp syndrome present late-onset renal disease $(25,26)$. It has been reported a 43 -year-old patient with the same variant of $M A F$ (p.Ser54Leu) presented with proteinuria and typical clinical features of Aymé-Gripp syndrome, subsequently diagnosed with mesangiocapillary glomerulopathy (26). Our findings suggest that renal manifestations of MPGN could also present in children with Aymé-Gripp syndrome and add clinical value in monitoring patients with $M A F$ pathogenic variants for changes in renal function.

A new proposed diagnostic standard of C3G was C3 dominant at least two orders of magnitude more intense than any other immune reactant, which requires validation by alternative pathway evaluation $(20,21)$. We applied this 
Table 3 Summary of correlated factors with clinical remission

\begin{tabular}{|c|c|c|c|c|}
\hline Variables & Total & $\begin{array}{l}\text { Complete or partial } \\
\text { remission }\end{array}$ & Non-response & $P$ value \\
\hline Number & 17 & 8 & 9 & / \\
\hline Prednisolone & 15 & 7 & 8 & 1.000 \\
\hline MMF & 7 & 3 & 4 & 1.000 \\
\hline CTX & 5 & 1 & 4 & 0.294 \\
\hline ACEI & 10 & 7 & 3 & 0.05 \\
\hline Age onset (years) & $9.9(5.6,11.9)$ & $10.8(6.4,12.0)$ & $8.2(5.4,11.0)$ & 0.309 \\
\hline Follow-up (years) & $2.4(1.0,4.5)$ & $1.6(0.3,2.4)$ & $3.6(2.1,8.2)$ & 0.023 \\
\hline Initial eGFR (mL/min/1.73 m²) & $102.6(47.0,121.5)$ & $106.8(103.0,126.4)$ & $47.0(35.5,109.1)$ & 0.092 \\
\hline $\begin{array}{l}\text { Initial proteinuria ( } 24 \text { hours urine } \\
\text { protein quantification) }\end{array}$ & $2.6(1.5,5.4)$ & $2.7(1.8,3.8)$ & $2.5(1.0,7.2)$ & 0.378 \\
\hline Primary MPGN & 9 & 6 & 3 & 0.153 \\
\hline $\begin{array}{l}\text { Other renal/extrarenal } \\
\text { manifestations }\end{array}$ & 3 & 0 & 3 & 0.206 \\
\hline
\end{tabular}

eGFR was calculated by the Schwartz formula using a local k-factor of 49 in CKD 1-2, 36.5 in CKD 3-5. Data are given as median (interquartile range). ACEI, angiotensin converting enzyme inhibitor; CTX, Cyclophosphamide; MMF, mycophenolate mofetil; MPGN, membranoproliferative glomerulonephritis.

standard in our pediatric patients and found two patients with obvious alternative pathway evaluation would be failed to identify the diagnosis of C3G in this condition. Similar situations were also observed in other pediatric MPGN studies $(15,16)$. Accordingly, there do exist several pediatric MPGN patients with dysregulation of alternative pathway who do not conform to the "C3 dominant at least two orders of magnitude more intense than any other immune reactant" criterion. It seems that current diagnostic criteria of IF labeling for $\mathrm{C} 3 \mathrm{G}$ may be strict on pediatric patients with MPGN. Previous studies had shown the prognosis of patients with $\mathrm{C} 3 \mathrm{G}$ is worse than patients with IC-MPGN, particularly in DDD patients $(23,27)$. Therefore, we must take a close look at the clinical course for the patients with C3 dominant deposit in the glomerulus. Outcome-related factors were also analyzed in this study. We discovered most patients who received early diagnosis reached complete or partial remission at the last follow-up, whereas patients who received delayed diagnosis all turned out non-response for usual treatments. It suggests that early pathological diagnosis in children may help to optimize the treatment and prevent the decline of renal function. Although there are controversial results, it has recently been shown that the treatment of MPGN with corticosteroids plus MMF 


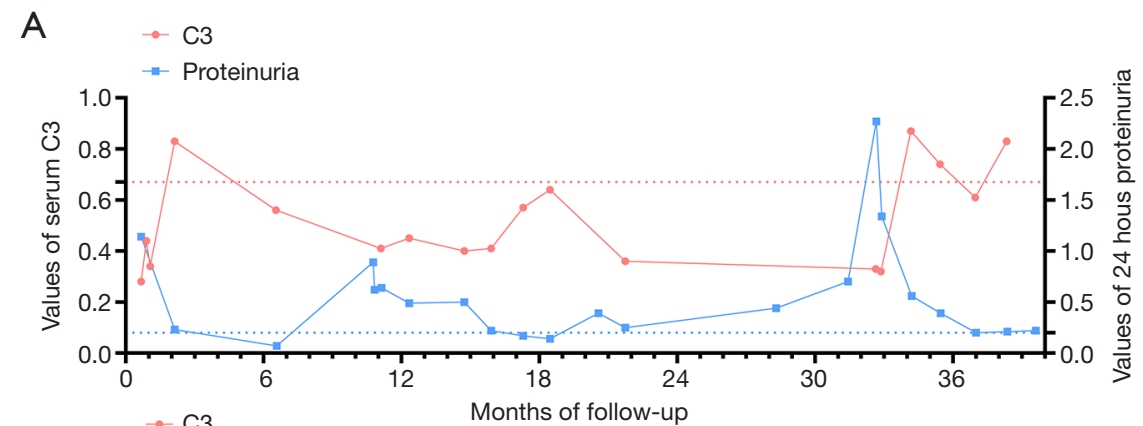

C

-- Proteinuria

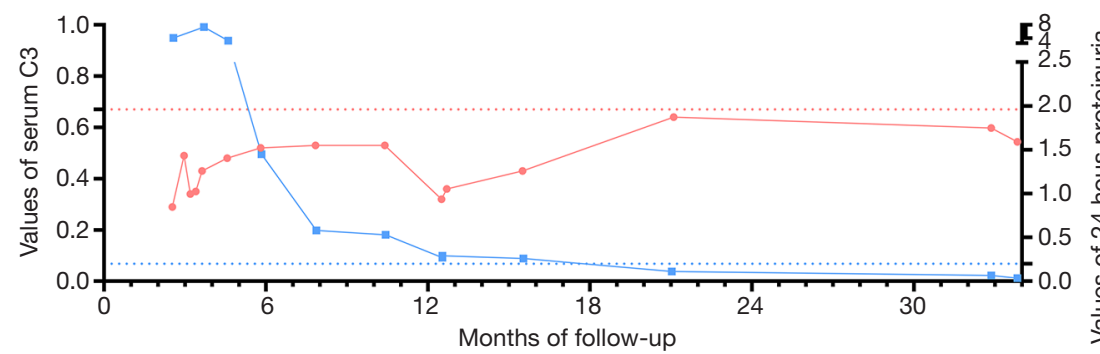

B

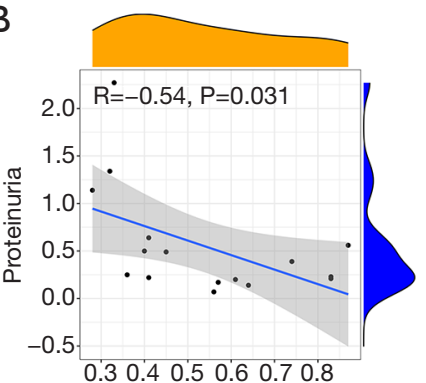

D

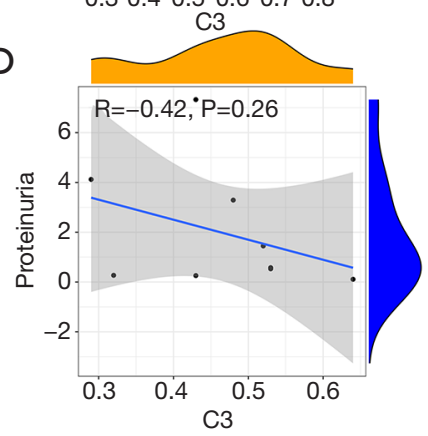

Figure 4 Evolution of serum C3 and proteinuria in two patients with initial positive C3NeF. P value was analyzed by Spearman correlation. $(A, B)$ Correlation analysis of serum C3 with proteinuria in patient \#3. (C,D) Correlation analysis of serum C3 with proteinuria in patient \#14.

in adults caused better kidney survival as compared with patients treated with other immunosuppressants and untreated patients (28).

Limitations of our study include the following: First, as a retrospective observational study, we were unable to perform genetic and serological tests in all patients, and therefore, evaluation of the possible effects of specific genetic or serological markers was not possible. Second, our study was small case series from a single-center study collecting the clinical data from 2007 to 2020. MPGN is rare, with an incidence estimated at 1-2 per million population (29). A large pediatric cohort of MPGN should be carried out in multiply medical centers from different regions. Third, long-term follow-up needs to be performed in this rare disease.

\section{Conclusions}

We present detailed valuable information on the pediatric case series of MPGN. Integrated analysis of genotype and phenotype including pathological and clinical findings is pivotal for early identification of the primary or secondary causes of MPGN in children.

\section{Acknowledgments}

Funding: JR is supported by a grant from National Natural Science Foundation of China (NSFC-8182207), a grant from Program of Shanghai Academic/Technology Research Leader (19XD1420600).

\section{Footnote}

Reporting Checklist: The authors have completed the STROBE reporting checklist. Available at https://dx.doi. org/10.21037/tp-21-286

Data Sharing Statement: Available at https://dx.doi. org/10.21037/tp-21-286

Peer Review File: Available at https://dx.doi.org/10.21037/ tp-21-286

Conflicts of Interest: All authors have completed the ICMJE uniform disclosure form (available at https://dx.doi. org/10.21037/tp-21-286). The authors have no conflicts of interest to declare. 
Ethical Statement: The authors are accountable for all aspects of the work in ensuring that questions related to the accuracy or integrity of any part of the work are appropriately investigated and resolved. The study was conducted in accordance with the Declaration of Helsinki (as revised in 2013). The study was approved by Institutional Review Board (IRB) of Children's Hospital of Fudan University (No. 2018_286) and informed consent was taken from all the patients' parents.

Open Access Statement: This is an Open Access article distributed in accordance with the Creative Commons Attribution-NonCommercial-NoDerivs 4.0 International License (CC BY-NC-ND 4.0), which permits the noncommercial replication and distribution of the article with the strict proviso that no changes or edits are made and the original work is properly cited (including links to both the formal publication through the relevant DOI and the license). See: https://creativecommons.org/ licenses/by-nc-nd/4.0/.

\section{References}

1. Swaminathan S, Leung N, Lager DJ, et al. Changing incidence of glomerular disease in Olmsted County, Minnesota: a 30-year renal biopsy study. Clin J Am Soc Nephrol 2006;1:483-7.

2. Zhou FD, Zhao MH, Zou WZ, et al. The changing spectrum of primary glomerular diseases within 15 years: a survey of 3331 patients in a single Chinese centre. Nephrol Dial Transplant 2009;24:870-6.

3. Briganti EM, Dowling J, Finlay M, et al. The incidence of biopsy-proven glomerulonephritis in Australia. Nephrol Dial Transplant 2001;16:1364-7.

4. O'Shaughnessy MM, Hogan SL, Poulton CJ, et al. Temporal and Demographic Trends in Glomerular Disease Epidemiology in the Southeastern United States, 19862015. Clin J Am Soc Nephrol 2017;12:614-23.

5. Heaf JG, Sørensen SS, Hansen A. Increased incidence and improved prognosis of glomerulonephritis: a national 30year study. Clin Kidney J 2020;14:1594-602.

6. Smith RJ, Alexander J, Barlow PN, et al. New approaches to the treatment of dense deposit disease. J Am Soc Nephrol 2007;18:2447-56.

7. Sethi S, Fervenza FC. Membranoproliferative glomerulonephritis--a new look at an old entity. N Engl J Med 2012;366:1119-31.

8. Sethi S, Fervenza FC. Membranoproliferative glomerulonephritis: pathogenetic heterogeneity and proposal for a new classification. Semin Nephrol 2011;31:341-8.

9. Masani N, Jhaveri KD, Fishbane S. Update on membranoproliferative GN. Clin J Am Soc Nephrol 2014;9:600-8.

10. Nakano M, Karasawa K, Moriyama T, et al. Characteristics of membranoproliferative glomerulonephritis based on a new classification at a single center. Clin Exp Nephrol 2019;23:852-8.

11. Wilson GJ, Cho Y, Teixiera-Pinto A, et al. Long-term outcomes of patients with end-stage kidney disease due to membranoproliferative glomerulonephritis: an ANZDATA registry study. BMC Nephrol 2019;20:417.

12. Nakagawa N, Hasebe N, Hattori M, et al. Clinical features and pathogenesis of membranoproliferative glomerulonephritis: a nationwide analysis of the Japan renal biopsy registry from 2007 to 2015. Clin Exp Nephrol 2018;22:797-807.

13. Iatropoulos $P$, Daina $E$, Curreri $M$, et al. Cluster Analysis Identifies Distinct Pathogenetic Patterns in C3 Glomerulopathies/Immune Complex-Mediated Membranoproliferative GN. J Am Soc Nephrol 2018;29:283-94.

14. Lu Y, Shen P, Li X, et al. Re-evaluation of the classification system for membranoproliferative glomerulonephritis. Contrib Nephrol 2013;181:175-84.

15. Holle J, Berenberg-Goßler L, Wu K, et al. Outcome of membranoproliferative glomerulonephritis and C3glomerulopathy in children and adolescents. Pediatr Nephrol 2018;33:2289-98.

16. Spartà G, Gaspert A, Neuhaus TJ, et al. Membranoproliferative glomerulonephritis and C3 glomerulopathy in children: change in treatment modality? A report of a case series. Clin Kidney J 2018;11:479-90.

17. Okuda Y, Ishikura K, Hamada R, et al.

Membranoproliferative glomerulonephritis and C3 glomerulonephritis: frequency, clinical features, and outcome in children. Nephrology (Carlton) 2015;20:286-92.

18. Radhakrishnan J, Cattran DC. The KDIGO practice guideline on glomerulonephritis: reading between the (guide)lines--application to the individual patient. Kidney Int 2012;82:840-56.

19. Floege J, Barbour SJ, Cattran DC, et al. Management and treatment of glomerular diseases (part 1): conclusions from a Kidney Disease: Improving Global Outcomes (KDIGO) Controversies Conference. Kidney Int 2019;95:268-80. 
20. Pickering MC, D'Agati VD, Nester CM, et al. C3 glomerulopathy: consensus report. Kidney Int 2013;84:1079-89.

21. Hou J, Markowitz GS, Bomback AS, et al. Toward a working definition of $\mathrm{C} 3$ glomerulopathy by immunofluorescence. Kidney Int 2014;85:450-6.

22. Çaltik Yilmaz A, Aydog Ö, Akyüz SG, et al. The relation between treatment and prognosis of childhood membranoproliferative glomerulonephritis. Ren Fail 2014;36:1221-5.

23. Khandelwal P, Bhardwaj S, Singh G, et al. Therapy and outcomes of C3 glomerulopathy and immune-complex membranoproliferative glomerulonephritis. Pediatr Nephrol 2021;36:591-600.

24. Levine AP, Chan MMY, Sadeghi-Alavijeh O, et al. LargeScale Whole-Genome Sequencing Reveals the Genetic Architecture of Primary Membranoproliferative GN and C3 Glomerulopathy. J Am Soc Nephrol 2020;31:365-73.

25. Alkhunaizi E, Koenekoop RK, Saint-Martin C, et al.

Cite this article as: $\mathrm{Xu} \mathrm{L}$, Wei F, Feng J, Liu J, Liu J, Tang X, Fang X, Chen J, Zhai Y, Liu H, Sun L, Qian Y, Wu B, Wang H, Shen Q, Rao J, Xu H. Characteristics and outcomes of glomerulonephritis with membranoproliferative pattern in children. Transl Pediatr 2021;10(11):2985-2996. doi: 10.21037/ tp-21-286
Maternally inherited MAF variant associated with variable expression of Aymé-Gripp syndrome. Am J Med Genet A 2019;179:2233-6.

26. Niceta M, Stellacci E, Gripp KW, et al. Mutations Impairing GSK3-Mediated MAF Phosphorylation Cause Cataract, Deafness, Intellectual Disability, Seizures, and a Down Syndrome-like Facies. Am J Hum Genet 2015;96:816-25.

27. Kawasaki Y, Kanno S, Ono A, et al. Differences in clinical findings, pathology, and outcomes between C3 glomerulonephritis and membranoproliferative glomerulonephritis. Pediatr Nephrol 2016;31:1091-9.

28. Kirpalani A, Jawa N, Smoyer WE, et al. Long-Term Outcomes of C3 Glomerulopathy and Immune-Complex Membranoproliferative Glomerulonephritis in Children. Kidney Int Rep 2020;5:2313-24.

29. McGrogan A, Franssen CF, de Vries CS. The incidence of primary glomerulonephritis worldwide: a systematic review of the literature. Nephrol Dial Transplant 2011;26:414-30. 
Table S1 Base line clinical characterizations of the patients with MPGN

\begin{tabular}{|c|c|c|c|c|c|c|c|c|c|c|c|c|}
\hline $\begin{array}{l}\text { Patient } \\
\text { No. }\end{array}$ & $\begin{array}{l}\text { Age }(y) / \\
\text { Sex }\end{array}$ & $\begin{array}{c}\text { Prior } \\
\text { infection }\end{array}$ & Edema & Hypertension & $\begin{array}{c}\text { Hematuria (/ } \\
\text { HPF) }\end{array}$ & $\begin{array}{l}\text { 24-hour urinary } \\
\text { protein (g) }\end{array}$ & $\mathrm{P} / \mathrm{C}$ & $\begin{array}{l}\text { Lipstick of } \\
\text { proteinuria }\end{array}$ & $\begin{array}{c}\text { Serum albumin } \\
(g / L)\end{array}$ & $\begin{array}{l}\text { Other renal/extrarenal } \\
\text { manifestations }\end{array}$ & Positive family history & LM/EM \\
\hline 1 & 9/F & $(-)$ & $(+)$ & $(-)$ & $(-)$ & 8.53 & NA & $3+$ & $\downarrow^{\mathrm{a}}$ & & & $\begin{array}{l}\text { Glomeruli hyalinized, mesangial and endocapillary proliferation, } \\
\text { crescents, tubular atrophy/NA }\end{array}$ \\
\hline 2 & $4 / \mathrm{M}$ & Dermal & $(+)$ & $(-)$ & 71.3 & 2.57 & 8.92 & $3+$ & 27.2 & & & $\begin{array}{l}\text { Mesangial and endocapillary proliferation, individual crescents, } 10 \% \\
\text { glomeruli hyalinized, tubular atrophy/Mild mesangial electron-dense } \\
\text { deposits }\end{array}$ \\
\hline 3 & $5 / \mathrm{M}$ & Dermal & $(-)$ & $(-)$ & 135 & 1.53 & 2 & $2+$ & 30.8 & & & $\begin{array}{l}\text { Mesangial and endocapillary proliferation, mild interstitial fibrosis, } \\
\text { segmental sclerosis, individual crescents, double contours of GBM/ } \\
\text { Mesangial, subendothelial and rare subepithelial electron-dense } \\
\text { deposits }\end{array}$ \\
\hline 5 & $6 / \mathrm{M}$ & $(-)$ & $(-)$ & $(-)$ & 12.32 & 0.26 & 0.81 & $2+$ & 43.8 & $\begin{array}{l}\text { Cataract; intellectual disability; } \\
\text { hearing impairment }\end{array}$ & & $\begin{array}{l}\text { Mesangial and endocapillary proliferation, interstitial fibrosis/Renal } \\
\text { afferent arteriolar dense deposits }\end{array}$ \\
\hline 6 & $4 / \mathrm{M}$ & $(-)$ & $(-)$ & $(+)$ & 67 & 0.54 & 3.95 & $3+$ & 40.5 & Hearing impairment & $\begin{array}{l}\text { The elder sister: diagnosed kidney } \\
\text { disease at } 4 \text { years old and deceased } \\
\text { at 6; Mother: SLE }\end{array}$ & $\begin{array}{l}\text { Mesangial and endocapillary proliferation, individual crescents/ } \\
\text { Subepithelial and rare subendothelial electron-dense deposits }\end{array}$ \\
\hline 7 & $11 / F$ & Respiratory & $(-)$ & $(-)$ & 195 & 0.8 & 1.06 & $3+$ & 18.4 & & & Mesangial proliferation/NA \\
\hline 8 & $10 / \mathrm{M}$ & Dermal & $(-)$ & $(-)$ & 35 & 2.54 & 1.42 & $3+$ & 26.4 & & $\begin{array}{l}\text { Parents: recurrent urticaria; Cousins: } \\
\text { history of NS or hematuria }\end{array}$ & $\begin{array}{l}\text { Mesangial and endocapillary proliferation, mild interstitial fibrosis, } \\
\text { double contours of GBM /No observed electron-dense deposits }\end{array}$ \\
\hline 10 & $10 / F$ & $(-)$ & $(+)$ & $(-)$ & 50 & 2.6 & NA & $2+$ & $\downarrow^{\circ}$ & & & $\begin{array}{l}\text { Mesangial and endocapillary proliferation, interstitial fibrosis /Mesangial } \\
\text { and subepithelial electron-dense deposits }\end{array}$ \\
\hline 11 & $4 / \mathrm{M}$ & Dermal & $(-)$ & $(-)$ & 6 & 5.9 & NA & $3+$ & Normal $^{d}$ & Renal malrotation (Right) & $\begin{array}{l}\text { Aunt and uncle: history of edema and } \\
\text { proteinuria; Uncle: diabetes insipidus }\end{array}$ & $\begin{array}{l}\text { Mesangial and endocapillary proliferation, double contours of GBM, } \\
\text { glomeruli hyalinized, interstitial fibrosis/NA }\end{array}$ \\
\hline 12 & $11 / \mathrm{F}$ & $(-)$ & $(+)$ & $(-)$ & 70 & 2.78 & NA & $2+$ & $\downarrow^{e}$ & & & $\begin{array}{l}\text { Mesangial and endocapillary proliferation, double contours of GBM, } \\
\text { tubular atrophy/NA }\end{array}$ \\
\hline 13 & $12 / \mathrm{F}$ & $(-)$ & $(-)$ & $(+)$ & 7 & 2.53 & 2.18 & $3+$ & 35.5 & & & $\begin{array}{l}\text { Segmental sclerosis, mesangial and endocapillary proliferation, } \\
\text { crescents, tubular atrophy, interstitial fibrosis, glomeruli hyalinized/No } \\
\text { observed electron-dense deposits }\end{array}$ \\
\hline 14 & $12 / \mathrm{F}$ & Respiratory & $(+)$ & $(+)$ & 8 & 4.17 & 4.34 & $3+$ & 23.9 & & & $\begin{array}{l}\text { Mesangial and endocapillary proliferation/Mild mesangial and } \\
\text { subendothelial electron-dense deposits }\end{array}$ \\
\hline 15 & 9/F & Respiratory & $(+)$ & $(-)$ & $(-)$ & 14.9 & NA & $3+$ & $\downarrow^{t}$ & & & Mesangial and endocapillary proliferation, tubular atrophy/NA \\
\hline 16 & $12 / \mathrm{M}$ & $(-)$ & $(+)$ & $(-)$ & 20 & 4.88 & NA & $3+$ & 31 & & & $\begin{array}{l}\text { Crescents, mesangial and endocapillary proliferation, segmental } \\
\text { sclerosis, tubular atrophy, interstitial fibrosis/Severe subendothelial and } \\
\text { subepithelial electron-dense deposits }\end{array}$ \\
\hline 17 & $7 / \mathrm{M}$ & $(-)$ & $(-)$ & $(-)$ & $\infty$ & 1.5 & NA & $2+$ & NA & & & $\begin{array}{l}\text { Mesangial and endocapillary proliferation, crescents, tubular atrophy, / } \\
\text { Mild mesangial electron-dense deposits }\end{array}$ \\
\hline
\end{tabular}

$10,11,12$ and 15 were evaluated by serum protein electrophoresis. $33 \%$ (a), $59.9 \%$ (b), $55.1 \%$ (c), $66.7 \%$ (d), $52.8 \%$ (e) and $58.4 \%$ (f) 
Table S2 Clinical characteristics and laboratory parameters of patients with MPGN at last follow-up

\begin{tabular}{|c|c|c|c|c|c|c|c|c|}
\hline Patient No. & Complications & Hematuria(/HPF) & eGFR(ml/min·1.73m²) & $\begin{array}{l}\text { 24-hour urinary } \\
\text { protein (g) }\end{array}$ & $\begin{array}{l}\text { Lipstick of } \\
\text { proteinuria }\end{array}$ & C3 (g/L) & C3 $\mathrm{NeF}$ & $\begin{array}{l}\text { Time from disease onset to } \\
\text { renal biopsy (months) }\end{array}$ \\
\hline 1 & Hypertension dental ulcers & $(-)$ & 5.9 & 4.8 & $2+$ & NA & NA & 19.6 \\
\hline 2 & dental ulcers & 72.18 & 148.7 & NA & $1+$ & NA & NA & 0.6 \\
\hline 3 & Hypertension & 63.39 & 109.5 & 0.11 & $(-)$ & 0.83 & $(-)$ & 1.3 \\
\hline 4 & Hypertension & 21.55 & 110.3 & NA & micro & 1.1 & NA & 1.8 \\
\hline 5 & $(-)$ & $(-)$ & 190.9 & NA & $1+$ & NA & NA & 1.7 \\
\hline 6 & $(-)$ & $(-)$ & 66.1 & NA & $2+$ & NA & NA & 1.2 \\
\hline 7 & $(-)$ & $(-)$ & 148.0 & NA & + & 1.65 & NA & 0.3 \\
\hline 8 & $(-)$ & 90 & 115.1 & 1.08 & $3+$ & 0.1 & NA & 13.1 \\
\hline 9 & $(-)$ & $(-)$ & 134.9 & 0.49 & $(-)$ & NA & NA & 4.4 \\
\hline 10 & $(-)$ & $(-)$ & 104.0 & NA & $(-)$ & NA & NA & 2.0 \\
\hline 11 & $(-)$ & $(-)$ & 33.9 & NA & $2+$ & NA & NA & 84.4 \\
\hline 12 & $(-)$ & $(-)$ & 110.0 & NA & $(-)$ & NA & NA & 1.6 \\
\hline 13 & $(-)$ & $(-)$ & 5.6 & NA & NA & NA & NA & 7.3 \\
\hline 14 & $\begin{array}{l}\text { High intraocular pressure } \\
\text { Binocular cataract Urinary } \\
\text { tract infection }\end{array}$ & $(-)$ & 135.2 & 0.11 & $(-)$ & 0.64 & $(-)$ & 2.6 \\
\hline 15 & Urinary tract infection & $(-)$ & 70.8 & $(-)$ & $(-)$ & NA & NA & 15.4 \\
\hline 16 & Urinary tract infection & $(-)$ & NA & NA & $3+$ & NA & NA & 7.1 \\
\hline 17 & blurred vision & $(-)$ & 75.4 & NA & $(-)$ & NA & NA & 63.7 \\
\hline
\end{tabular}

A

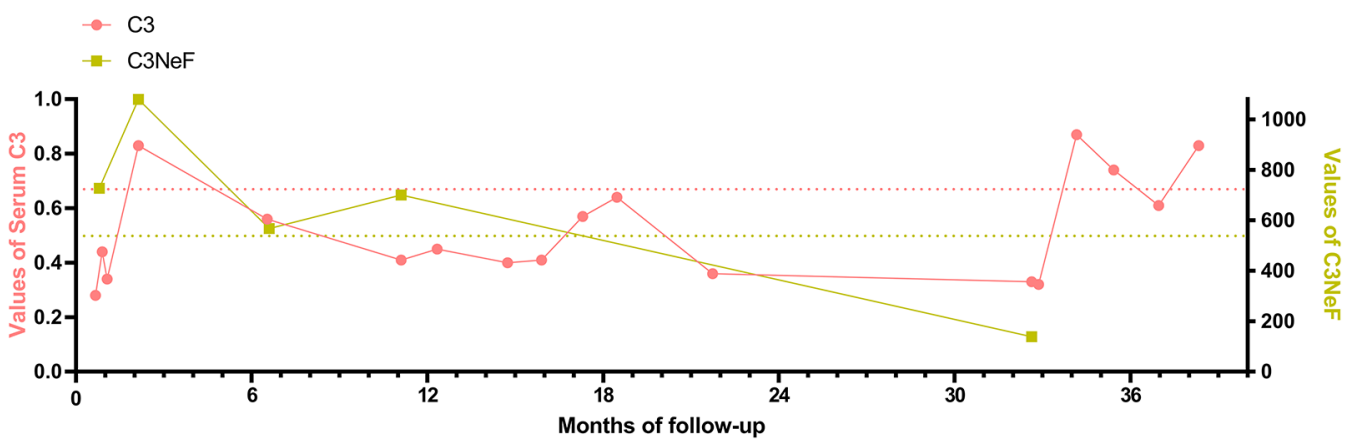

$\mathrm{B} \rightarrow \mathrm{C} 3$

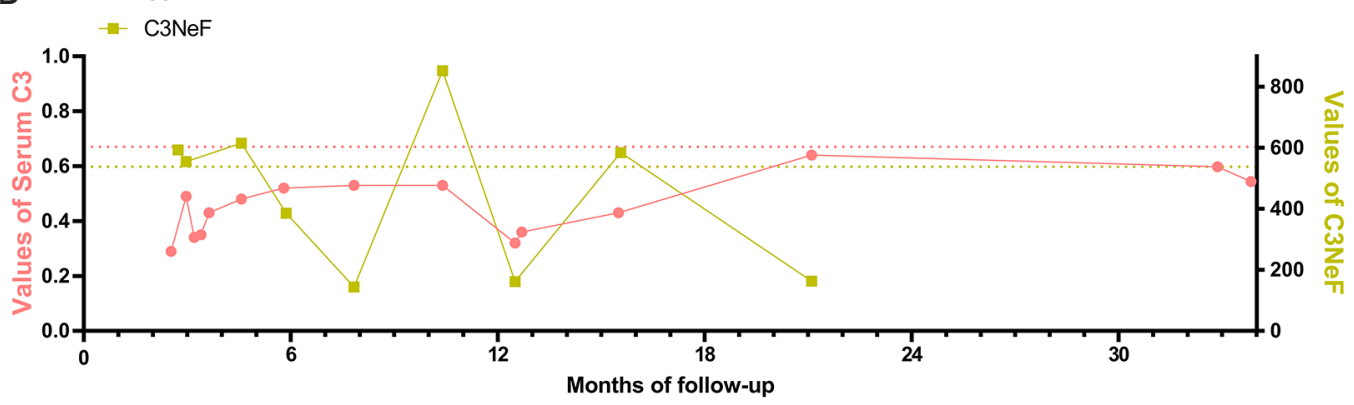

Figure S1 Evolution of serum C3 and C3NeF in the whole course of disease. (A) patient 3; (B) patient 14. 